\title{
Real-time Observation on Coarsening of Second-Phase Droplets in Al-Bi Immiscible Alloy Using Synchrotron Radiation X-ray Imaging Technology
}

\author{
Hui-Jun Kang ${ }^{1} \cdot$ Peng Zhou ${ }^{1} \cdot$ Fei Cao ${ }^{1} \cdot J^{3} g \mathrm{Zhu}^{2} \cdot$ Ya-Nan Fu ${ }^{3} \cdot$ \\ Wan-Xia Huang $^{4}$ - Ti-Qiao Xiao ${ }^{3}$ - Tong-Min Wang ${ }^{1}$
}

Received: 4 February 2015/Revised: 9 April 2015/Published online: 13 May 2015

(c) The Chinese Society for Metals and Springer-Verlag Berlin Heidelberg 2015

\begin{abstract}
The coarsening process of second-phase droplet in solidifying Al-20 wt $\%$ Bi immiscible alloy is in situ studied using synchrotron radiation imaging technology. The collision-induced coarsening and Ostwald coarsening phenomena are directly observed and analyzed. It is found that through observation, collision-induced coarsening phenomenon occurs between droplets with little difference in radius, while Ostwald coarsening phenomenon occurs among droplets with much difference in radius. Moreover, the coarsening rate of Ostwald coarsening is much higher than that of collision-induced coarsening.
\end{abstract}

\section{KEY WORDS: Al alloys; Coarsening; Secondary phase; Droplet particles}

\section{Introduction}

Immiscible alloys exhibit unique properties including superb plasticity and anti-attrition properties due to the special distribution of second-phase droplets in the matrix. They are widely used as important structural and functional materials, such as advanced bearings in automotive applications, self-lubricating materials, electrical contact materials and superconducting materials [1-7]. Experimental results indicate that mechanical properties of the

Available online at http://link.springer.com/journal/40195

Tong-Min Wang

tmwang@dlut.edu.cn

1 Key Laboratory of Materials Modification By Laser, Ion and Electron Beams (Ministry of Education), School of Materials Science and Engineering, Dalian University of Technology, Dalian 116024, China

2 Dalian Marine Propeller Co. Ltd, Dalian 116021, China

3 Shanghai Institute of Applied Physics, Chinese Academy of Sciences, Shanghai 201204, China

4 Institute of High Energy Physics, Chinese Academy of Sciences, Beijing 100039, China immiscible alloys are generally determined by the distribution of second phases of droplets in the matrix $[8,9]$. In previous investigations [10-14], it was found that coarsening phenomenon resulted from coagulation and collision coalescences frequently occurs during solidification due to the effect of Stokes sedimentation and Marangoni migration in the melt, which is definitely detrimental to the tribological property of immiscible alloys as advanced bearing materials. However, as a typical immiscible alloy, the $\mathrm{Al}-\mathrm{Bi}$ alloy is considered to be one of the most promising materials as advanced bearings and superconducting materials [2-7]. Therefore, in order to improve the mechanical properties of the Al-Bi immiscible alloys, it is very important to figure out the coarsening mechanism of the second-phase droplets during solidification.

In recent years, much work has been done on the coarsening mechanism of second-phase droplets in immiscible alloys under microgravity and gravity [15-19]. It has been found that Marangoni force has a significant effect on the motion and coarsening of second-phase droplets [5, 10-12, 20-22]. In the last decade, synchrotron radiation imaging technology, which provides a real-time study of the liquid metals during solidification, has become one of the most advanced methods for material science. The growth of 
metal alloy crystals has been in situ observed using synchrotron radiation source together with an X-ray chargecoupled device (CCD) camera [23-28]. Synchrotron radiation imaging technology was used by Mathiesen to observe the directional solidification process of immiscible alloys. It was found that large droplets were frequently pushed by monotectic front as soft particles while small droplets were engulfed, and droplet-droplet interaction and local solute gradients are critical for the pushing/engulfment behavior of droplets in Al-Bi immiscible alloys [29]. Nevertheless, the coarsening mechanism of second-phase droplets in Al-Bi immiscible alloys is important but still remains unclear. The objective of this paper is to dynamically observe the formation, dissociation and coarsening phenomenon of the second-phase droplets and quantitatively measure the coarsening rate in $\mathrm{Al}-20 \mathrm{wt} \% \mathrm{Bi}$ immiscible alloy for a better understanding of the Stokes sedimentation, Marangoni migration and the dynamical behavior of the collision coalescences. This work also provides a direct proof of the theoretical model for liquid-liquid phase change/dissociation in $\mathrm{Al}-\mathrm{Bi}$ immiscible alloys.

\section{Experimental}

$\mathrm{Al}-20 \mathrm{wt} \% \mathrm{Bi}$ alloy was prepared by melting $\mathrm{Al}$ and $\mathrm{Bi}$ with a purity of $99.99 \%$ in a graphite crucible. In order to make the $\mathrm{Al}-\mathrm{Bi}$ alloy homogeneous, the melt was kept in the resistance furnace for 10-15 min with stirring. Considering the $\mathrm{Al}-\mathrm{Bi}$ master alloy was kind of layered structure, the samples were chosen using the bottom of the Al-Bi ingot due to its good foundry quality. The samples were then cut into rectangular sheets with dimensions of $20 \mathrm{~mm} \times 10 \mathrm{~mm} \times$ $200 \mu \mathrm{m}$. Those sheets were sandwiched between two ceramic plates enclosed with gypsum. A $100-\mu \mathrm{m}$-thick mica sheet was also placed between two ceramic plates to fix the alloy sample. Moreover, clips were used to pinch the two ceramic chips to keep the sample still during the whole imaging process. Then the sheet sample was melted using specially designed double furnaces for the synchrotron microradiography experiment. Each furnace consists of heating coils wrapped with heat insulation fiber-cotton. The temperature of each furnace (hot and cold furnaces) could be separately controlled by a thyristor controller to monitor the variations of temperature, as shown in Fig. 1. There is a window in the middle of the furnace for X-ray to pass through the sample. An X-ray CCD camera was placed behind the furnace to dynamically capture the time-sequenced images.

The experiments were carried out at the beamline BL13W1 of Shanghai Synchrotron Radiation Facility (SSRF) in China, which is a third-generation synchrotron radiation facility. The imaging method was coaxial phase-

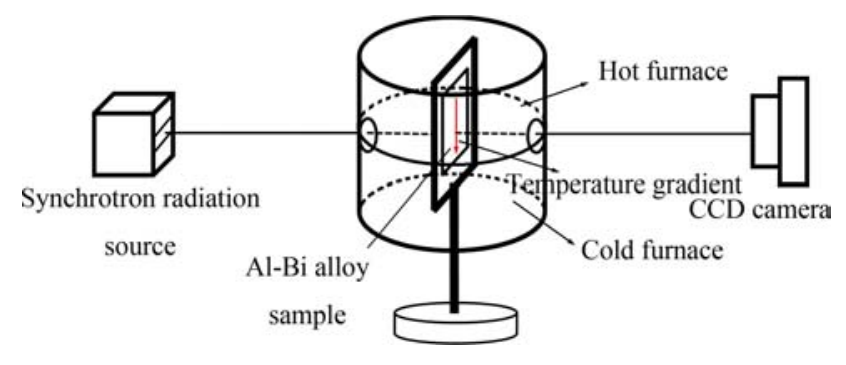

Fig. 1 Schematic diagram of experimental setup

contrasting imaging method. During the experiment, the prepared sample was firstly put into the furnace to completely melt at $1273 \mathrm{~K}$ with a temperature gradient of about $6 \mathrm{~K} / \mathrm{cm}$, which was monitored by controlling the temperatures of hot and cold furnaces. The temperature gradient, which is considered to have a great effect on the solidification of the melt, is marked on the sample in Fig. 1. Temperatures in both hot and cold furnaces were then synchronously decreased at a cooling rate of $5 \mathrm{~K} / \mathrm{min}$ during solidification. The CCD camera has a rectangular view of $2048 \times 2048$ pixel array, and its pixel size is down to $3.7 \mu \mathrm{m}$. The sample-to-detector distance is $15-20 \mathrm{~cm}$. The exposure time and the energy of the X-ray beam are $2 \mathrm{~s}$ and $18 \mathrm{keV}$, respectively, which are quite adequate to capture the details during solidification.

\section{Results and Discussion}

\subsection{Collision-induced Coarsening and Ostwald Coarsening Phenomena}

Figure 2 shows a sequence of six images at different time during solidification with a cooling rate of $5 \mathrm{~K} / \mathrm{min}$. The image sequence here represents the initial stage $\left(t_{0}\right)$, which is just after the solidification of Bi-rich droplets begins. Two Bi-rich droplets that emerge as two black spots are highlighted by the arrows and noted as A and B in Fig. 2a. Droplet $\mathrm{B}$ gradually grows in size and approaches to droplet A with increasing time from $t_{0}$ to $t_{0}+457 \mathrm{~s}$ (Fig. $2 \mathrm{~b}$, c). Droplet B grows to a radius of $61.3 \mu \mathrm{m}$ (measured by Image-Pro Plus software) and collides with droplet $\mathrm{A}$ at $t_{0}+466 \mathrm{~s}$ (Fig. 2d). Finally, those two droplets coagulate into a nearly elliptical droplet and the coagulated droplet gradually deposits, leading to a process of collision-induced coarsening (Fig. 2e, f).

Figure 3 displays another coarsening process of Bi-rich droplets. Core-type droplet $\mathrm{C}$ (highlighted by a circle in Fig. 3a) forms during the liquid-liquid phase separation, and it is surrounded by a large number of Bi-rich droplets with a tiny radius size. During the solidification, small droplets which are adjacent to $\mathrm{C}$ droplet disappear little by 

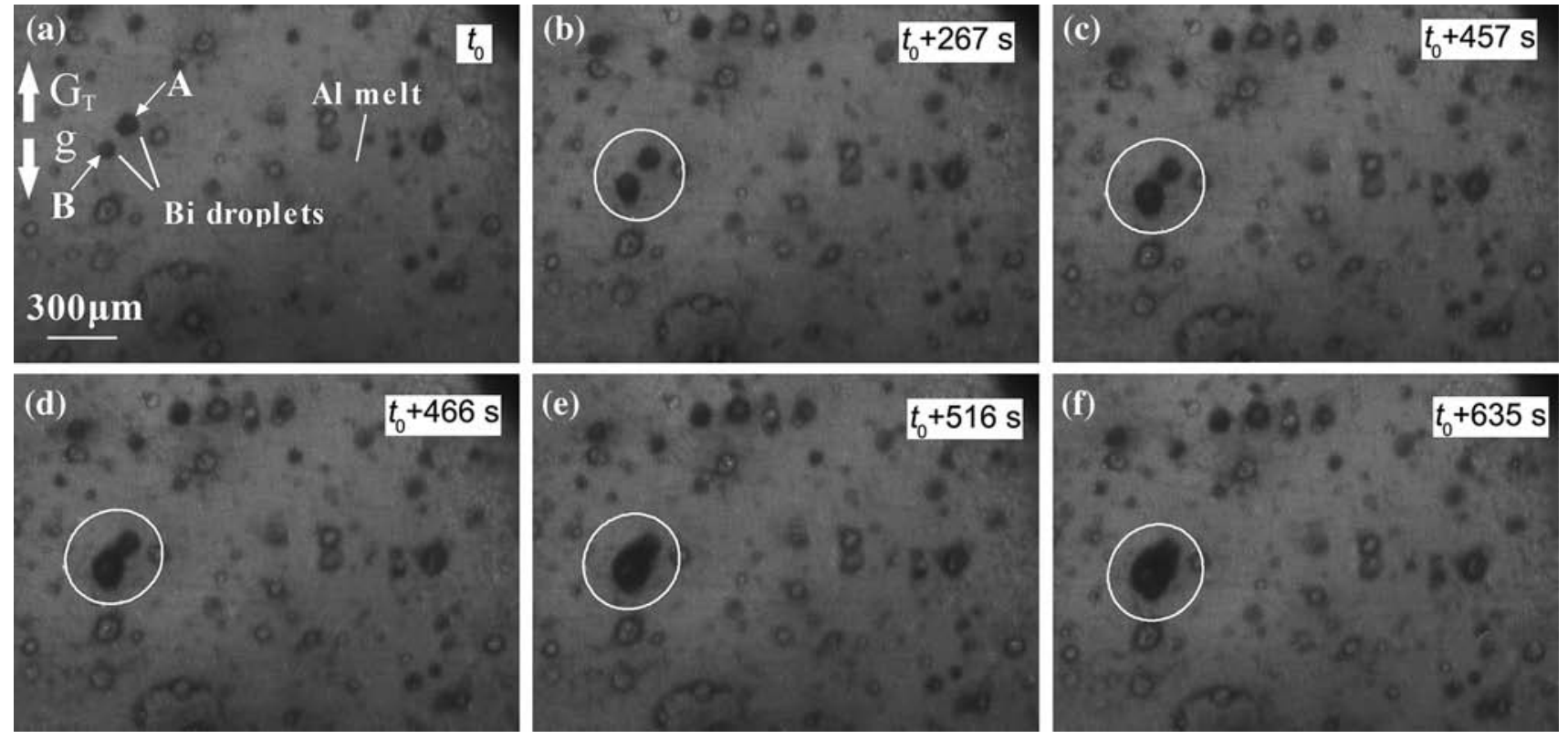

Fig. 2 Collision-induced coarsening process of Bi-rich droplets with a field of view of $1.88 \mathrm{~mm} \times 1.29 \mathrm{~mm}: \mathbf{a} t_{0}$, b $t_{0}+267 \mathrm{~s}$, c $t_{0}+457 \mathrm{~s}$, d $t_{0}+466 \mathrm{~s}$, e $t_{0}+516 \mathrm{~s}, \mathbf{f} t_{0}+635 \mathrm{~s}$

little, and the radius of droplet $\mathrm{C}$ increases as time goes on. It is thought that those small droplets are coalesced by $\mathrm{C}$ droplet. This kind of coarsening process is considered to be Ostwald coarsening.

\subsection{Growth of the Droplets and Their Moving Behaviors}

The distance between the center of the droplet and the bottom boundary of the frame (droplet height) is quantitatively measured by Image-Pro Plus software. The data are sequentially illustrated in Figs. 4 and 5. Each droplet (A, B and C) is selected as a typical one in the frame for measurement. In Fig. 4, the radius of droplet A remains constant before $t_{0}+516 \mathrm{~s}$, and the radius of droplet $\mathrm{B}$ continuously increases by diffusion growth. Droplet A is trammeled on the ceramic plate in the early stage of liquid-liquid phase separation process. Consequently, it does not grow anymore and its radius remains constant. Droplet $B$ is just separated from the liquid phase and it grows up rapidly during the period of diffusion growth. Therefore, the radius of droplet $\mathrm{B}$ obviously increases with the increase in time. It can be seen from Fig. 4 that there is a striking difference in radius between the conditions that are with or without trammel. Collision happens and a bigger droplet forms while droplet B approaches to droplet A during the growing process. The radius of the newly formed droplet suddenly increases from 41 to $71 \mu \mathrm{m}$, which is considered to be the biggest droplet. Here, the radii of $r_{1}=41 \mu \mathrm{m}$ and $r_{2}=71 \mu \mathrm{m}$ are defined as the initial size and the final size in the whole coarsening process, and the time duration is set as $\Delta t$. Thus, the coarsening rate $v_{0}$ of the coagulated droplet is calculated by $v_{0}=\frac{r_{2}-r_{1}}{\Delta t}$ and approximately $0.067 \mu \mathrm{m} / \mathrm{s}$. It can be seen from Fig. $4 \mathrm{~b}$ that in the initial stage of solidification, the droplets deposit separately due to the density difference of the melt, which shortens the droplet height. At $t_{0}+466 \mathrm{~s}$, droplet $B$ has a tiny increase in size as the result of its colliding with droplet A during the growing process. The reason can be explained as follows: During the movement of the droplet, the gravity of the droplet is higher than the Marangoni force. Thus, the droplet tends to move downward due to the downward direction of the resultant force. However, when the two droplets begin to coalesce, directional adsorption effect is dominant for the two droplets rather than the gravity or the Marangoni force, which undoubtedly forces the droplets to attract each other. That is why droplet B has a tiny increase in size before coalescence, as shown in Fig. 4. When droplet A and droplet B collide and start to coalesce, a nearly spherical shape eventually forms through simple growth [30]. After the coalescence event, the newly formed droplet changes its shape with time because the coalesced droplet ceases to grow for a period of time. As the liquid-liquid phase separation proceeds, the occupancy volume of $\mathrm{Al}$ melt gradually decreases so that it is difficult for the coalesced droplet to move after the relaxation period expires. Therefore, in the final stage of solidification, the droplet becomes nearly elliptical rather than spherical. 

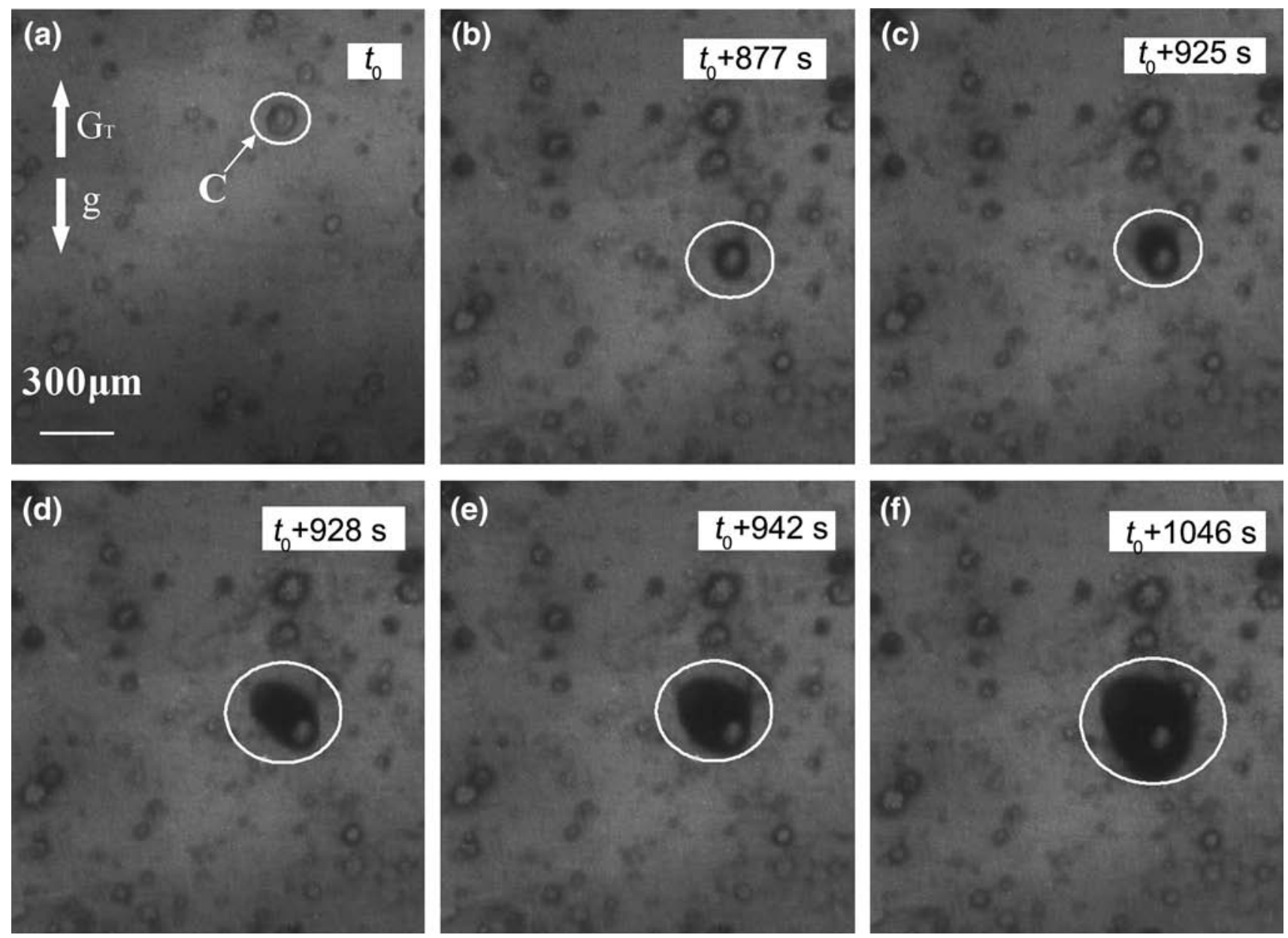

Fig. 3 Ostwald coarsening process of Bi-rich droplets with a field of view of $1.21 \mathrm{~mm} \times 1.29 \mathrm{~mm}: \mathbf{a} t_{0}, \mathbf{b} t_{0}+877 \mathrm{~s}, \mathbf{c} t_{0}+925 \mathrm{~s}$, d $t_{0}+928 \mathrm{~s}, \mathbf{e} t_{0}+942 \mathrm{~s}, \mathbf{f} t_{0}+1046 \mathrm{~s}$

On the other hand, as shown in Fig. 5a, the radius size of droplet $\mathrm{C}$ increases slowly through diffusion growth in the early stage of solidification. From $t_{0}+877 \mathrm{~s}$ to $t_{0}+1046 \mathrm{~s}$, the radius of droplet $\mathrm{C}$ increases at a certain growth rate $v_{1}$. Here, the initial radius and final radius of droplet $\mathrm{C}$ are denoted as $r_{3}$ and $r_{4}$. Thus, the growth rate can be calculated as $v_{1}=\frac{r_{4}-r_{3}}{\Delta t}=\frac{r_{4}-r_{3}}{169}=1.82 \mu \mathrm{m} / \mathrm{s}$. In this stage, there are a great number of small droplets with tiny radius around droplet $\mathrm{C}$. Those small droplets are gradually engulfed by droplet $\mathrm{C}$ due to the less supersaturation in the melt. Consequently, droplet $\mathrm{C}$ becomes a nearly rounded droplet with a huge radius in a way of snowballing growth (from $t_{0}+877 \mathrm{~s}$ to $t_{0}+1046 \mathrm{~s}$ ). This process is regarded as Ostwald coarsening process. As shown in Fig. 5b, droplet $\mathrm{C}$ gradually comes down to the bottom due to the effect of Stokes flow from $t_{0}$ to $t_{0}+877 \mathrm{~s}$. Consequently, the settling rate markedly decreases and the Ostwald coarsening starts because of the increasing viscosity of the semisolid Al melt.

\subsection{Two Models of Coarsening Phenomena}

In order to compare the two coarsening processes mentioned above, two models have been proposed in Fig. 6 . Model I represents the collision-induced coarsening phenomenon, which mainly occurs among droplets that are mostly the same in radius. The growth of the two droplets is separately controlled by their diffusion behavior in the initial stage of solidification. Afterward, droplet B begins to grow up and gradually approaches to droplet $\mathrm{A}$ and collides with droplet $\mathrm{A}$ in the intermediate stage of solidification. The surface tension induced by the contractive surface in the melt drives the two droplets to coagulate so that a new elliptical droplet appears in the final stage of solidification. Model II reveals the Ostwald coarsening phenomenon, which occurs among droplets with huge differences in radius. All the droplets grow through diffusion-controlled growth behavior that is similar to that of Model I in the initial stage of solidification. In the 


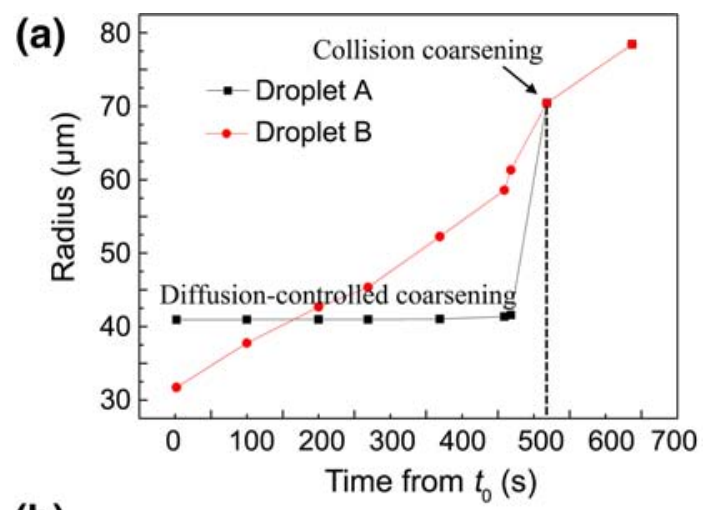

(b)

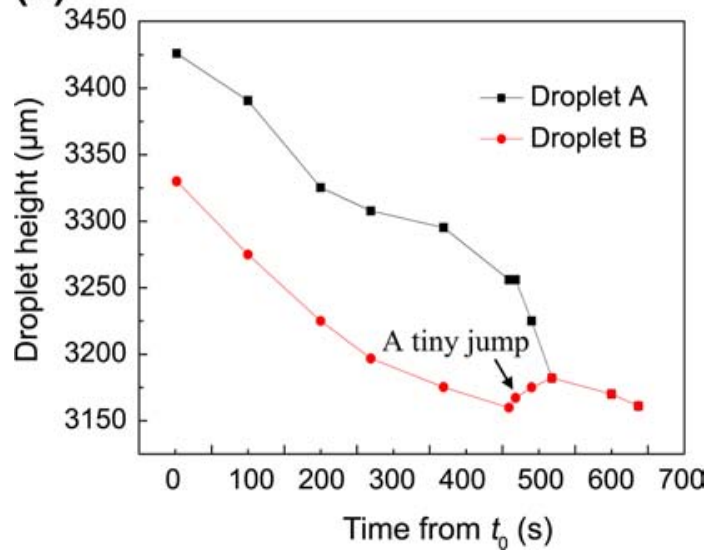

Fig. 4 Variation of radius and motion trajectories of droplets A and $\mathrm{B}$ with time: a droplet radius, $\mathbf{b}$ motion trajectories

intermediate stage of solidification, the droplets around droplet $\mathrm{C}$ with tiny radius size begin to pile up on the surface of droplet $C$ layer upon layer. As a result, droplet $C$ engulfs them little by little and ends up in a bigger one. Finally, the smaller droplets are swallowed by droplet $C$ in the supersaturated melt, which leads to the expansion of droplet $\mathrm{C}$ to a nearly spherical droplet with a tremendous radius. In the previous studies [31, 32], an evolutional process of core-shell microstructures and the phase field of monotectic transformation for liquid $\mathrm{Ni}$ and $\mathrm{Ni}-\mathrm{Cu}-\mathrm{Pb}$ alloys have been simulated, which reveals that those two models are acceptable in general.

\section{Conclusions}

It can be concluded that two kinds of dynamic coarsening processes of second-phase droplets in $\mathrm{Al}-\mathrm{Bi}$ immiscible alloy have been in situ observed using synchrotron radiation imaging technique. The collision-induced coarsening and Ostwald coarsening are directly observed and analyzed in detail. Both of them can be divided into three stages: initial stage, intermediate stage and final stage. The initial stages of the two cases are the same with a diffusion-
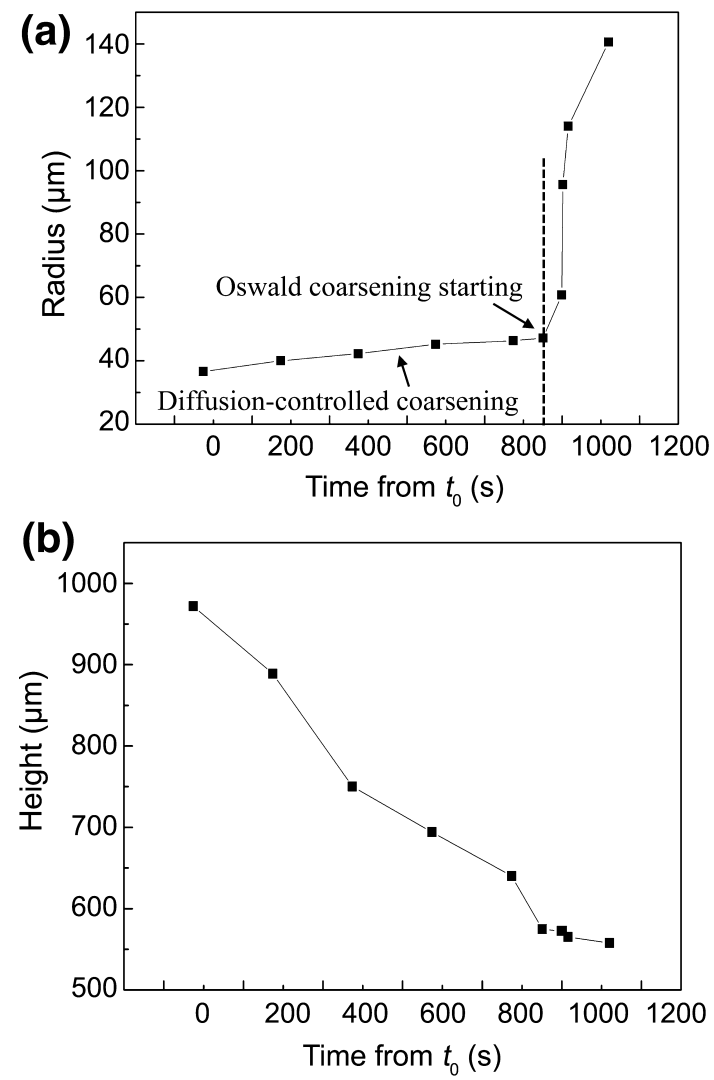

Fig. 5 Variation in radius and motion trajectories of droplet $\mathrm{C}$ with time: a droplet radius, $\mathbf{b}$ motion trajectories

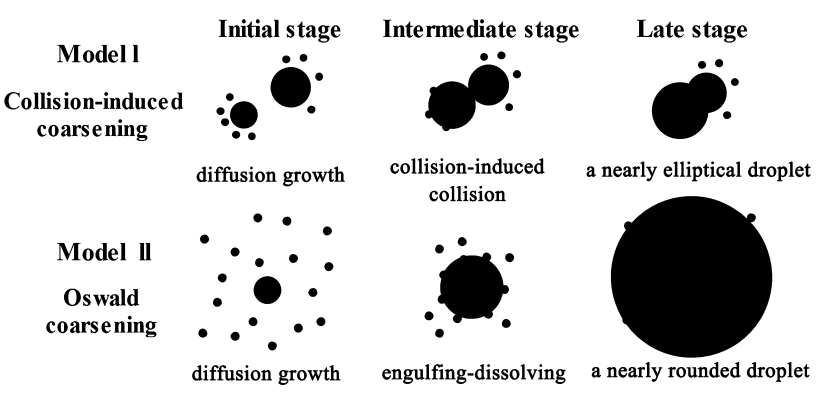

Fig. 6 Schematic representation of two coarsening models among droplets

controlled growth behavior. For collision-induced coarsening, the intermediate stage is the collision phenomenon between two droplets with similar radii, while for Ostwald coarsening, a big droplet engulfs a large number of small droplets around it in the intermediate stage. A nearly elliptical droplet and a nearly spherical droplet are finally formed in the final stages of the two cases, respectively. The mechanisms are analyzed based on the interface tension and dissolving theories. Therefore, it is concluded that synchrotron radiation imaging technology can be used to in situ visualize the coarsening process of second-phase 
droplets in $\mathrm{Al}-\mathrm{Bi}$ immiscible alloy to capture the direct evidence and kinetic data in this paper.

Acknowledgments This work was financially supported by the National Natural Science Foundation of China (Nos. 51274054, U1332115, 51271042, 51375070 and 51401044), the Keygrant Project of Chinese Ministry of Education (No. 313011) and the China Postdoctoral Science Foundation (2014M551075). The authors wish to thank all the staff members of the BL13W1 beamline of SSRF and 4W1A beamline of Beijing Synchrotron Radiation Facility (BSRF) for corresponding experiments and discussion.

\section{References}

[1] A. Berkdemir, M. Gündüz, Appl. Phys. A 96, 873 (2009)

[2] W.R. Osório, E.S. Freitas, A. Garcia, Electrochim. Acta 108, 781 (2013)

[3] T.M. Wang, J. Zhu, H.J. Kang, Z.N. Chen, Y.N. Fu, W.X. Huang, T.Q. Xiao, Appl. Phys. A 117, 1059 (2014)

[4] G.J. Feng, Z.R. Li, R.H. Liu, S.C. Feng, Acta Metall. Sin. (Engl. Lett.) 28, 405 (2015)

[5] X.F. Li, W. Ding, J. Cao, L.Y. Ye, J. Chen, Acta Metall. Sin. (Engl. Lett.) 28, 302 (2015)

[6] J. Chang, H.P. Wang, K. Zhou, B. Wei, Appl. Phys. A 109, 139 (2012)

[7] J.Z. Zhao, H.L. Li, H.Q. Li, C.Y. Xing, X.F. Zhang, Q.L. Wang, J. He, Comp. Mater. Sci. 49, 121 (2010)

[8] A.P. Silva, A. Garcia, J.E. Spinelli, J. Mater. Sci. Technol. 30, 401 (2014)

[9] A.P. Silva, J.E. Spinelli, N. Mangelinck-Noël, A. Garcia, Mater. Des. 31, 4584 (2010)

[10] W.L. Wang, Z.Q. Li, B. Wei, Acta Mater. 59, 5482 (2011)

[11] R.P. Shi, Y. Wang, C.P. Wang, X.J. Liu, Appl. Phys. Lett. 98, 204106 (2011)
[12] C.P. Wang, X.J. Liu, I. Ohnuma, Science 297, 990 (2002)

[13] J.Z. Zhao, L. Ratke, Scr. Mater. 50, 543 (2004)

[14] J.Z. Zhao, Scr. Mater. 54, 247 (2006)

[15] T. Carlberg, H. Fredriksson, Metall. Trans. A 11, 1665 (1980)

[16] J.R. Rogers, R.H. Davis, Metall. Trans. A 21, 59 (1990)

[17] S. Ozawa, T. Motegi, Mater. Lett. 58, 2548 (2004)

[18] M. Wu, A. Ludwig, L. Ratke, Metall. Mater. Trans. A 34, 3009 (2003)

[19] M.H. Wu, A. Ludwig, M. Pelzer, U. Postl, Adv. Eng. Mater. 7, 846 (2005)

[20] C. Marangoni, Ann. Phys. 219, 337 (1871)

[21] W. Lu, S. Zhang, J. Li, Mater. Lett. 107, 340 (2013)

[22] L. Ratke, S. Diefenbach, S. Drees, J. Alkemper, B. Prinz, A. Romero, H. Ahlborn, Adv. Space Res. 16, 185 (1995)

[23] A. Bogno, H. Nguyen-Thi, N. Bergeon, N. Mangelinck-Noël, T. Schenk, B. Billia, E. Boller, J. Baruchel, Nucl. Instrum. Methods Phys. Res. B 268, 394 (2010)

[24] A. Bogno, H. Nguyen-Thi, G. Reinhart, B. Billia, J. Baruchel, Acta Mater. 61, 1303 (2013)

[25] Q. Dong, J. Zhang, J. Dong, H. Xie, Z. Li, Y. Dai, Y. Liu, B. Sun, Mater. Sci. Eng. A 530, 271 (2011)

[26] H. Yasuda, Y. Yamamoto, N. Nakatsuka, T. Nagira, M. Yoshiya, A. Sugiyama, I. Ohnaka, K. Umetani, K. Uesugi, Int. J. Cast Metal Res. 21, 125 (2008)

[27] A.G. Murphy, D.J. Browne, W.U. Mirihanage, R.H. Mathiesen, Acta Mater. 61, 4559 (2013)

[28] H. Nguyen-Thi, G. Reinhart, A.J.G. Salloum, R.H. Mathiesen, G. Zimmermann, Y. Houltz, D. Voss, A. Verga, D.J. Browne, A.G. Murphy, J. Cryst. Growth 374, 23 (2013)

[29] P.L. Schaffer, R.H. Mathiesen, L. Arnberg, Acta Mater. 57, 2887 (2009)

[30] H. Zheng, R.K. Smith, Y. Jun, C. Kisielowski, U. Dahmen, A.P. Alivisatos, Science 324, 1309 (2009)

[31] T. Qin, H.P. Wang, B.B. Wei, Sci. China, Ser. G 50, 546 (2007)

[32] B.C. Luo, H.P. Wang, B.B. Wei, Chin. Sci. Bull. 54, 183 (2009) 